\title{
Therapeutic effect of irbesartan combined with atorvastatin calcium in the treatment of rats with coronary heart disease
}

\author{
TAO LI ${ }^{1}$ and WEINA YAO ${ }^{2}$ \\ ${ }^{1}$ Emergency Center, Jinan Zhangqiu District Hospital of TCM, Jinan, Shandong 250200; \\ ${ }^{2}$ Department of Cardiovascular Medicine, The First People's Hospital of Jinan, Jinan, Shandong 250000, P.R. China
}

Received March 2, 2018; Accepted August 6, 2018

DOI: $10.3892 /$ etm.2018.6669

\begin{abstract}
This study aimed to investigate the therapeutic effect of irbesartan combined with atorvastatin calcium in the treatment of rats with coronary heart disease. One hundred sixty Wistar rats were selected to establish coronary heart disease model. Rats with coronary heart disease were randomly divided into 4 groups: Model, irbesartan, atorvastatin calcium and combination groups (irbesartan combined with atorvastatin calcium group). Rats in irbesartan group were treated with $50 \mathrm{mg} /(\mathrm{kg}$.day) irbesartan; rats in atorvastatin calcium group were given atorvastatin calcium at a dose of $10 \mathrm{mg} /(\mathrm{kg} \cdot \mathrm{day})$; rats in combination group were subjected to atorvastatin calcium at a dose of $10 \mathrm{mg} /(\mathrm{kg} \cdot$ day $)$ and irbesartan at a dose of $50 \mathrm{mg} /(\mathrm{kg} \cdot \mathrm{day})$, while rats in model groups were given intragastric administration of normal saline at a dose of $2 \mathrm{ml} /$ day. Serum lipids, including total cholesterol (TC), triglyceride (TG), low-density lipoprotein cholesterol (LDLC), high-density lipoprotein cholesterol (HDL-C) and TC/ HDL-C, were measured by automatic biochemical analyzer. Expression of sPLA2-V in myocardium and aortic trunk of rats was detected by reverse transcription-PCR (RT-PCR) and western blot analysis. After treatment, levels of serum TC, TG, LDL-C, HDL-C and TC/HDL-C in rats of each treatment group were better than those in model group $(\mathrm{p}<0.05)$. Expression level of sPLA2-V in myocardium and aortic trunk in model group was significantly higher than that in other groups $(\mathrm{p}<0.05)$. Expression level of sPLA2-V in combination group was significantly lower than that in irbesartan and atorvastatin calcium groups $(\mathrm{p}<0.05)$. Combination of irbesartan and atorvastatin calcium is superior to irbesartan or atorvastatin calcium alone in the treatment of rats with coronary heart disease. The possible explanation is that the two drugs can reduce the expression of SPLA2-V in myocardium and aortic
\end{abstract}

Correspondence to: Dr Tao Li, Emergency Center, Jinan Zhangqiu District Hospital of TCM, 1463 Xiushui Avenue, Jinan, Shandong 250200, P.R. China

E-mail: tbm4e2@163.com; 1002598343@qq.com

Key words: irbesartan, atorvastatin calcium, coronary heart disease, combination therapy, sPLA2-V, atherosclerosis trunk, which in turn relieved atherosclerosis and achieved better therapeutic effect.

\section{Introduction}

Coronary heart disease is a common type of heart disease with high incidence. More than 20 million people die each year from cardiovascular disease, of which about $42 \%$ die of coronary heart disease (1). About $50 \%$ of people over the age of 50 suffer from coronary heart disease worldwide (2), and the incidence and mortality of coronary heart disease are still rising (3). Coronary heart disease has become the number one killer of human health. Current treatment of coronary heart disease mainly aims to control the disease, reduce complications, enhance postoperative care and reduce the number of treatment. Therefore, early diagnosis and treatment of coronary heart disease is the key to improve survival $(4,5)$.

At present, diagnosis of coronary heart disease is mainly based on dyslipidemia (6). Treatment methods mainly include interventional, surgical and drug treatments, of which drug treatment is particularly important (7). Statins are widely used in drug treatment and show promising therapeutic effects. Statins also play an important role in secondary prevention of coronary heart disease (8). Clinical studies have confirmed that atorvastatin has a satisfactory effect in the treatment of coronary heart disease $(9,10)$. In addition, therapeutic effect of angiotensin II receptor blocker (ARB) on coronary heart disease is also satisfactory. Irbesartan belongs to the ARB, and studies have shown that high-dose irbesartan can effectively reduce blood pressure, reduce the degree of carotid atherosclerosis, and relieve clinical symptoms (11). Type II secretory phospholipase A2 (sPLA2) is a member of the family of calcium-dependent phospholipases that is highly expressed in atherosclerotic lesions, and can affect atherosclerosis and lead to coronary heart disease (12). SPLA2-V as a subtype of sPLA2, and has been detected in atherosclerotic plaques (13). In the present study, irbesartan and atorvastatin calcium were used together to investigate their effects on sPLA2-V expression in myocardium and aorta in rats with coronary heart disease, and to explore the possible mechanism.

\section{Materials and methods}

Experimental animals. One hundred sixty healthy male Wistar rats, 3-4 months old, weighing approximately $200 \mathrm{~g}$, were 
provided by China Medical University (Taichung, Taiwan). The rats were kept in cages with controlled temperature and light cycles $\left(24^{\circ} \mathrm{C}\right.$ and $12 / 12$ light cycles) with free access to water and food. The humidity was $60 \pm 10 \%$. This study was approved by the Ethics Committee of Jinan Zhangqiu District Hospital of TCM (Jinan, China).

Drugs and major reagents. Irbesartan was purchased from Jiangsu Hengrui Medicine Co., Ltd. (state approval no. H20000513; Lianyungang, China). Atorvastatin calcium was purchased from Beijing Jialin Pharmaceutical Co., Ltd. (state approval no. H20093819; Beijing, China). TRIzol and RT-PCR kit were purchased from Sangon Biotech (Shanghai) Co., Ltd. (Shanghai, China). Total cholesterol (TC), triglyceride (TG), low-density lipoprotein cholesterol (LDL-C) and high-density lipoprotein cholesterol (HDL-C) kits (all from Cyttel Bioscience Inc., Beijing, China). Skim milk powder (BD Biosciences, San Jose, CA, USA), rabbit anti-rat sPLA2-V polyclonal antibody (cat no. 16009-1-AP; $1: 1,000)$ and goat anti-rabbit-HRP secondary polyclonal antibody (cat. no. SA00001-2; 1:800) were purchased from Wuhan Sanying Biotechnology, Wuhan, China. Protein electrophoresis buffer, transfer membrane buffer and washing, all from Sangon Biotech (Shanghai) Co., Ltd.

Model construction. The 160 healthy rats were fed with high-fat diet ( $2 \%$ cholesterol, $10 \%$ lard, $0.5 \%$ sodium cholate, $87.3 \%$ basal diet) daily for 6 weeks, followed by intraperitoneal injection of pituitrin at a dose of $30 \mathrm{U} / \mathrm{kg}$ for 3 consecutive days. All rats were subjected to electrocardiogram and lipids test and 152 rats showed myocardial ischemia and hyperlipidemia, which are the symptoms of coronary heart disease, considering the model was constructed successfully.

Animal grouping and treatment. Rats with coronary heart disease were randomly divided into 4 groups to receive corresponding treatment, 38 rats in each group. Rats in irbesartan group were treated with $50 \mathrm{mg} /(\mathrm{kg} \cdot$ day $)$ irbesartan through intragastric administration. Rats in atorvastatin calcium group were given intragastric administration of atorvastatin calcium at a dose of $10 \mathrm{mg} /(\mathrm{kg} \cdot$ day $)$. Rats in combination group were subjected to intragastric administration of atorvastatin calcium at a dose of $10 \mathrm{mg} /(\mathrm{kg} \cdot$ day $)$ and irbesartan at a dose of $50 \mathrm{mg} /(\mathrm{kg} \cdot$ day $)$, while rats in model groups were given intragastric administration of normal saline at a dose of $2 \mathrm{ml} / \mathrm{day}$. Treatment was performed for 12 weeks.

\section{Detection indicators}

Collection of specimens. After intragastric administration for 12 weeks, rats in each group were fasted for $16 \mathrm{~h}$ and anesthetized by intraperitoneal injection of $10 \%$ chloral hydrate. Chest was opened, and heart was punctured to collect blood, followed by centrifugation at 8,000 x $\mathrm{g}$ for $15 \mathrm{~min}$ to prepare serum. Heart and aortic trunk were dissected, and the right apex of left ventricular myocardium was taken, rinsed with pre-cooling $0.9 \%$ saline, and then placed in $4 \%$ paraformaldehyde solution for fixation.

Automatic biochemical analyzer was used to measure TC, TG, LDL-C, HDL-C and TC/HDL-C according to the instructions of corresponding kit (Cyttel Bioscience Inc.).
Table I. Primer sequences used in PCR reaction.

\begin{tabular}{llc}
\hline \multicolumn{1}{l}{ Genes } & \multicolumn{1}{c}{ Primers sequence } & $\begin{array}{c}\text { Length of } \\
\text { product (bp) }\end{array}$ \\
\hline sPLA2-V F: 5'-TCACGCTGGCTTGGTTCCTG-3' & 320 \\
R: 5'-CAATCATGGACTTCAGTTCT-3' & \\
GAPDH & F: 5'-TGACTCCACTCACGGCAAATTCAA-3' & 580 \\
R: 5'-CTAGTTGAATGCTTGGATGTACAA-3' & \\
\hline
\end{tabular}

sPLA2-V, type II secretory phospholipase A2-V.

RT-PCR to detect the expression of SPLA2-V. TRIzol was used to extract total RNA from the apex and aorta, and cDNA was synthesized by using RNA as template through reverse transcription according to the instructions of the kit, and $1 \mu \mathrm{l}$ of cDNA was used as template in PCR amplification (Table I). Reaction conditions were: $95^{\circ} \mathrm{C}$ for $10 \mathrm{~min} ; 95^{\circ} \mathrm{C}$ for $50 \mathrm{sec}$, followed by 30 cycles of $56^{\circ} \mathrm{C}$ for $50 \mathrm{sec}$ and $72^{\circ} \mathrm{C}$ for $60 \mathrm{sec}$, and $72^{\circ} \mathrm{C}$ for $10 \mathrm{~min}$. PCR products were detected by $1.5 \%$ agarose gel electrophoresis. GAPDH was used as endogenous control to normalize the intensity of each band to reflect the relative expression level of each gene.

Detection of SPLA2-V protein expression. The preserved myocardial and aortic specimens was mixed with lysis solution $(1 \mathrm{ml} / 100 \mathrm{mg})$, homogenized on ice, and then transferred to a centrifuge tube, followed by centrifugation at $3,000 \mathrm{xg}$ for $8 \mathrm{~min}$ to collect supernatant. Protein $(20 \mu \mathrm{g})$ was mixed with equal volume of $2 \mathrm{X}$ loading buffer and boiled in water for $5 \mathrm{~min}$, followed by electrophoresis under $100 \mathrm{~V}$ until the dye moved to the lower $1 / 3$ of the gel. Protein was transferred to PVDF membrane under $110 \mathrm{~mA}$ for $2 \mathrm{~h}$. Then membranes were blocked with $5 \%$ skimmed milk powder, followed by incubation with rabbit anti-rat SPLA2-polyclonal antibody overnight. The next day, membranes were washed 3 times, 5 min for each time, followed by incubation with goat anti-rabbit-HRP secondary polyclonal antibody at room temperature for $1 \mathrm{~h}$. Membranes were then washed 3 times, 5 min for each time, followed by addition of chemiluminescence substrate. Signals were detected by gel imager (Thermo Fisher Scientific, Inc., Waltham, MA, USA).

Statistical analysis. Statistical analysis was performed by using SPSS 17.0 (SPSS, Inc., Chicago, IL, USA). Count data were analyzed by $\chi^{2}$ test. Measurement data were expressed as the mean $\pm \mathrm{SD}$, and analysis of variance was used for comparisons among multiple groups and the post-hoc test was Least Significant Difference test. $\mathrm{P}<0.05$ was considered to indicate a statistically significant difference.

\section{Results}

Comparison of blood lipid indicators before treatment. Rats were randomly divided into different groups after model construction. There was no statistical difference in body weight among groups. Before treatment, no significantly differences in levels of TC, TG, LDL-C, HDL-C and TC/HDL-C were found among irbesartan, atorvastatin calcium, combination and model groups ( $\mathrm{p}>0.05)$ (Table II). 
Table II. Comparison of TC, TG, LDL-C, HDL-C and TC/HDL-C among groups before treatment (mean \pm SD).

\begin{tabular}{|c|c|c|c|c|c|c|}
\hline Groups $(n=38)$ & Model group & Irbesartan group & Atorvastatin calcium & Combination group & F-value & P-value \\
\hline Weight (g) & $601.52 \pm 28.52$ & $603.35 \pm 30.33$ & $603.98 \pm 30.15$ & $602.82 \pm 30.03$ & 0.004 & 1.000 \\
\hline $\mathrm{TC}(\mathrm{mmol} / \mathrm{l})$ & $5.01 \pm 1.04$ & $5.03 \pm 1.12$ & $5.05 \pm 1.03$ & $5.04 \pm 1.08$ & 0.001 & 0.435 \\
\hline $\mathrm{TG}(\mathrm{mmol} / \mathrm{l})$ & $2.26 \pm 0.26$ & $2.27 \pm 0.28$ & $2.23 \pm 0.25$ & $2.24 \pm 0.28$ & 0.014 & 0.996 \\
\hline LDL-C (mmol/l) & $3.09 \pm 0.30$ & $3.10 \pm 0.32$ & $3.11 \pm 0.33$ & $3.08 \pm 0.32$ & 0.005 & 0.999 \\
\hline HDL-C (mmol/l) & $1.17 \pm 0.24$ & $1.16 \pm 0.22$ & $1.13 \pm 0.24$ & $1.15 \pm 0.21$ & 0.017 & 0.389 \\
\hline TC/HDL-C (mmol/l) & $4.40 \pm 0.88$ & $4.42 \pm 0.90$ & $4.43 \pm 0.93$ & $4.40 \pm 0.87$ & 0.001 & 0.679 \\
\hline
\end{tabular}

TC, total cholesterol; TG, triglyceride; LDL-C, low-density lipoprotein cholesterol; HDL-C, high-density lipoprotein cholesterol.

Table III. Comparison of TC, TG, LDL-C, HDL-C and TC/HDL-C among groups after treatment (mean \pm SD).

\begin{tabular}{|c|c|c|c|c|c|c|}
\hline Groups $(\mathrm{n}=38)$ & Model group & Irbesartan group & Atorvastatin calcium & Combination group & F-value & P-value \\
\hline $\mathrm{TC}(\mathrm{mmol} / \mathrm{l})$ & $5.03 \pm 1.02$ & $4.09 \pm 0.15^{\mathrm{a}}$ & $3.80 \pm 0.11^{\mathrm{a}}$ & $3.58 \pm 0.18^{\mathrm{b}}$ & 4.417 & 0.041 \\
\hline TG $(\mathrm{mmol} / \mathrm{l})$ & $2.27 \pm 0.28$ & $1.90 \pm 0.14^{\mathrm{a}}$ & $1.92 \pm 0.04^{\mathrm{a}}$ & $1.78 \pm 0.15^{\mathrm{b}}$ & 4.373 & 0.043 \\
\hline LDL-C (mmol/l) & $3.21 \pm 0.11$ & $2.98 \pm 0.09^{\mathrm{a}}$ & $2.98 \pm 0.15^{\mathrm{a}}$ & $2.54 \pm 0.13^{\mathrm{b}}$ & 5.085 & 0.029 \\
\hline HDL-C (mmol/l) & $1.15 \pm 0.26$ & $1.33 \pm 0.25^{\mathrm{a}}$ & $1.32 \pm 0.18^{\mathrm{a}}$ & $1.88 \pm 0.18^{\mathrm{b}}$ & 6.210 & 0.017 \\
\hline TC/HDL-C (mmol/l) & $4.43 \pm 0.38$ & $4.07 \pm 0.48^{\mathrm{a}}$ & $3.96 \pm 0.34^{\mathrm{a}}$ & $2.98 \pm 0.42^{\mathrm{b}}$ & 6.919 & 0.013 \\
\hline
\end{tabular}

${ }^{a}$ Compared with control group, $\mathrm{p}<0.05$; ${ }^{\mathrm{b}}$ compared with irbesartan group or atorvastatin calcium group, $\mathrm{p}<0.05$. TC, total cholesterol; TG, triglyceride; LDL-C, low-density lipoprotein cholesterol; HDL-C, high-density lipoprotein cholesterol.

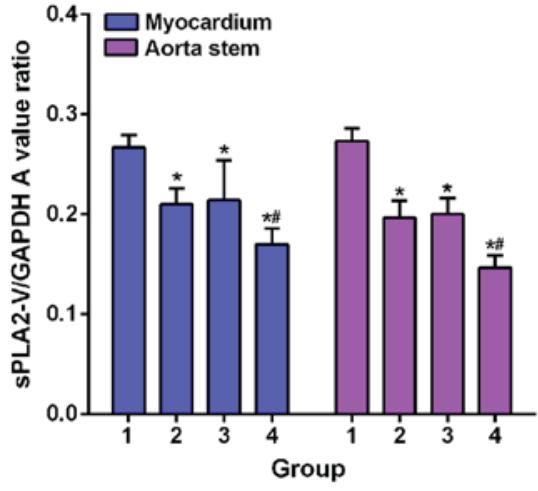

Figure 1. Comparisons of expression level of PLA2-V mRNA in myocardium and aortic trunk among groups. RT-PCR results showed that expression levels of sPLA2-V mRNA in apex and aorta of model groups (1) were significant higher than those of irbesartan group (2), atorvastatin calcium group (3) and combination group (4) ( $\mathrm{p}<0.05)$. In addition, expression levels of sPLA2-V mRNA in apex and aorta of combination group were significantly lower than those in irbesartan and atorvastatin calcium groups $\left({ }^{*}, \#<0.05\right)$. sPLA2-V, type II secretory phospholipase A2-V.

Comparison of blood lipid indicators after treatment. Levels of TC, TG, LDL-C, HDL-C and TC/HDL-C in rats of each group after 12 weeks of treatment were measured by automatic biochemical analyzer (Table III). Levels of TC, TG, LDL-C, and TC/HDL-C were significantly lower in irbesartan, atorvastatin calcium, and combination groups than those in model group $(\mathrm{p}<0.05)$. The decrease is more significant in combination group than that in irbesartan group or atorvastatin calcium group $(\mathrm{p}<0.05)$, while no significant difference was found between irbesartan and atorvastatin calcium groups. Levels of HDL-C were significantly higher in

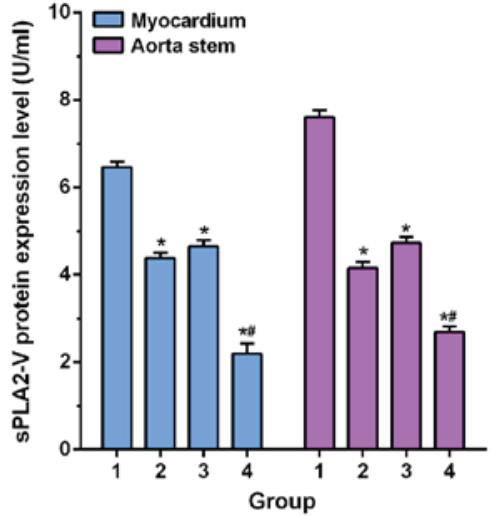

Figure 2. Comparisons of expression level of PLA2-V protein in myocardium and aortic trunk among groups. Western blot analysis results showed that expression levels of sPLA2-V protein in apex and aorta of model group (1) were significant higher than those of irbesartan group (2), atorvastatin calcium group (3) and combination group (4) $\left({ }^{*} \mathrm{p}<0.05\right)$. In addition, expression levels of sPLA2-V protein in apex and aorta of combination group were significantly lower than those in irbesartan and atorvastatin calcium groups $(*, \# p<0.05)$. sPLA2-V, type II secretory phospholipase A2-V.

irbesartan, atorvastatin calcium, and combination groups than in model group $(\mathrm{p}<0.05)$. The increase is more significant in combination group than in irbesartan or atorvastatin calcium group $(\mathrm{p}<0.05)$, while no significant difference was found between irbesartan and atorvastatin calcium groups.

Comparisons of expression level of PLA2-V mRNA in myocardium and aortic trunk among groups. GAPDH was as an endogenous control. sPLA2-V fragment was amplified by RT-PCR and analyzed by $1.5 \%$ agarose gel electrophoresis 
to determine the relative expression of sPLA2-V mRNA in each sample. After treatment, in 38 rats of irbesartan group, expression level of sPLA2-V in apex of heart was significantly decreased in 15 rats, and expression level of sPLA2-V in aorta was significantly decreased in 11 rats. In atorvastatin calcium group, expression level of sPLA2-V in apex of heart was significantly decreased in 19 rats, and expression level of sPLA2-V in aorta was significantly decreased in 15 rats. In combination group, expression level of sPLA2-V in apex of heart was significantly decreased in 30 rats, and expression level of sPLA2-V in aorta was also significantly decreased in 30 rats (Fig. 1). Expression levels of sPLA2-V mRNA in apex and aorta were the highest in model group. Expression levels of sPLA2-V mRNA in apex and aorta of model groups were significant higher than those of irbesartan, atorvastatin calcium and combination groups $(\mathrm{p}<0.05)$. In addition, expression levels of sPLA2-V mRNA in apex and aorta of combination group were significantly lower than those in irbesartan and atorvastatin calcium groups $(\mathrm{p}<0.05)$.

Comparisons of expression level of PLA2-V protein in myocardium and aortic trunk among groups. Western blot analysis results were shown in Fig. 2. Expression levels of sPLA2-V protein in apex and aorta of model group were significant higher than those of irbesartan, atorvastatin calcium and combination groups $(p<0.05)$. In addition, expression levels of sPLA2-V protein in apex and aorta of combination group were significantly lower than those in irbesartan and atorvastatin calcium groups $(\mathrm{p}<0.05)$.

\section{Discussion}

Coronary heart disease is a common disease endangering the health of the elderly, and the incidence is increasing year by year (14). Atherosclerosis can lead to organ disease (15). Occurrence of coronary heart disease may be related to dyslipidemia, genetic, environmental and other factors $(16,17)$.

At present, dyslipidemia is considered to be the pathological basis of atherosclerosis. TC and HDL-C play roles of promoting and inhibiting atherosclerosis respectively. TC/HDL-C ratio can reflect the balance between atherosclerosis and anti-atherosclerosis lipoproteins, and the status of cardiovascular events (18). In this study, Wistar rat model of coronary heart disease was established to explore the therapeutic effect of irbesartan and atorvastatin calcium on blood lipids. Results showed that all blood lipid indexes including TC, TG, HDL-C, LDL-C and TC/HDL-C were improved in irbesartan, atorvastatin calcium and combination groups compared with model group. Compared with irbesartan and atorvastatin calcium groups; TC, TG, LDL-C and TC/HDL-C decreased significantly, while HDL-C increased significantly in combination group, indicating that both irbesartan and atorvastatin calcium can effectively treat coronary heart disease, and the combined treatment is even better. Irbesartan can inhibit vasoconstriction, reduce the release of aldosterone, reduce blood pressure, and effectively treat coronary heart disease (19). In this study, irbesartan group also showed significantly improved blood lipid indexes, which is consistent with previous studies. Atorvastatin calcium can significantly reduce carotid atherosclerosis plaque in patients with ischemic attacks and improve blood lipid levels to achieve effective treatment of coronary heart disease (20). In this study, treatment with atorvastatin calcium significantly improved lipid indicators and reduced symptoms of coronary heart disease.

sPLA2-V is a subtype of sPLA2 that is mainly produced by vascular smooth muscle cells and macrophages. Higher expression level of sPLA2-V in atherosclerotic plaques than normal level indicates the risk of coronary heart disease (21). Irbesartan can inhibit atherosclerosis by reducing sPLA2-V expression $(22,23)$. In this study, sPLA2-V expression level in irbesartan group was significantly lower than that in model group, suggesting that irbesartan may inhibit the expression of sPLA2-V, thereby inhibiting the progression of atherosclerosis. Ikonomidis and Michalakeas (24) found that atorvastatin can reduce serum sPLA2IIa expression levels to achieve anti-atherosclerotic effect. In addition, irbesartan combined with revastatin can inhibit the expression of sPLA2-V (25), but the effects on irbesartan combined with atorvastatin calcium on expression of SPLA2-V remain unknown. In this study, atorvastatin calcium treatment effectively reduced SPLA2-V expression level in rats with heart disease, and the decrease was more significant in combination group than in irbesartan and atorvastatin calcium groups, while no significant differences were found between irbesartan and atorvastatin calcium groups, indicating that irbesartan combined with atorvastatin calcium is superior to irbesartan or atorvastatin calcium alone in inhibiting the expression of sPLA2-V.

In conclusion, therapeutic effect of combination of irbesartan and atorvastatin calcium is better than that of irbesartan or atorvastatin calcium alone in the treatment of rats with coronary heart disease. The possible explanation is that the two drugs can reduce the expression of SPLA2-V in myocardium and aortic trunk, which in turn relieved atherosclerosis and achieved better therapeutic effect.

\section{Acknowledgements}

Not applicable.

\section{Funding}

No funding was received.

\section{Availability of data and materials}

The datasets used and/or analyzed during the present study are available from the corresponding author on reasonable request.

\section{Authors' contributions}

TL was a major contributor in writing the manuscript, and designing the methods and the idea of this paper, and responsible for reviewing. WY participated in the analysis and discussion of the data, and responsible for the collection of the data and the follow-up management of the patients. All authors read and approved the final manuscript. 


\section{Ethics approval and consent to participate}

This study was approved by the Ethics Committee of Jinan Zhangqiu District Hospital of TCM (Jinan, China).

\section{Patient consent for publication}

Not applicable.

\section{Competing interests}

The authors declare that they have no competing interests.

\section{References}

1. Wang EY, Dixson J, Schiller NB and Whooley MA: Causes and predictors of death in patients with coronary heart disease (from the heart and soul study). Am J Cardiol 119: 27-34, 2017.

2. Woods SE: Primary prevention of coronary heart disease in women. Should asymptomatic women 50 years of age take aspirin regularly? Arch Fam Med 3: 361-364, 1994.

3. Menotti A, Puddu PE, Adachi H, Kafatos A, Tolonen $H$ and Kromhout D: The strength of the multivariable associations of major risk factors predicting coronary heart disease mortality is homogeneous across different areas of the Seven Countries Study during 50-year follow-up. Acta Cardiol 8: 1-7, 2017.

4. Brown JP, Clark AM, Dalal H, Welch K and Taylor RS: Patient education in the contemporary management of coronary heart disease. Cochrane Database Syst Rev 2010: CD008895, 2010.

5. Lebwohl B, Cao Y, Zong G, Hu FB, Green PHR, Neugut AI, Rimm EB, Sampson L, Dougherty LW, Giovannucci E, et al: Long term gluten consumption in adults without celiac disease and risk of coronary heart disease: Prospective cohort study. BMJ 357: j1892, 2017.

6. Lee JS, Chang PY, Zhang Y, Kizer JR, Best LG and Howard BV: Triglyceride and HDL-C dyslipidemia and risks of coronary heart disease and ischemic stroke by glycemic dysregulation status: The strong heart study. Diabetes Care 40: 529-537, 2017.

7. Lu W, Zhu Y, Han Z, Wang X, Wang X and Qiu C: Drug-coated balloon in combination with bare metal stent strategy for de novo coronary artery disease: A PRISMA-compliant meta-analysis of randomized clinical trials. Medicine (Baltimore) 96: e6397, 2017.

8. Serban MC, Muntner P and Rosenson RS: Reply: Statin intolerance and risk for recurrent myocardial infarction, Coronary heart disease events, and all-cause mortality. J Am Coll Cardiol 70: 685-686, 2017.

9. Wiklund O, Mattsson-Hultén L, Hurt-Camejo E and Oscarsson J: Effects of simvastatin and atorvastatin on inflammation markers in plasma. J Intern Med 251: 338-347, 2002.

10. Zhao Y, Peng R, Zhao W, Liu Q, Guo Y, Zhao S and Xu D: Zhibitai and low-dose atorvastatin reduce blood lipids and inflammation in patients with coronary artery disease. Medicine (Baltimore) 96: e6104, 2017.

11. Ricci F, De Caterina R and Fedorowski A: Orthostatic hypotension: Epidemiology, prognosis, and treatment. J Am Coll Cardiol 66: 848-860, 2015.

12. Liu PY, Li YH, Tsai WC, Chao TH, Tsai LM, Wu HL and Chen JH: Prognostic value and the changes of plasma levels of secretory type II phospholipase A2 in patients with coronary artery disease undergoing percutaneous coronary intervention. Eur Heart J 24: 1824-1832, 2003.
13. Holmes MV, Exeter HJ, Folkersen L, Nelson CP, Guardiola M, Cooper JA, Sofat R, Boekholdt SM, Khaw KT, Li KW, et al; CARDIoGRAM Consortium: Novel genetic approach to investigate the role of plasma secretory phospholipase A2 (sPLA2)-V isoenzyme in coronary heart disease: Modified Mendelian randomization analysis using PLA2G5 expression levels. Circ Cardiovasc Genet 7: 144-150, 2014.

14. Vaughan Dickson V, Lee CS, Yehle KS, Mola A, Faulkner KM and Riegel B: Psychometric testing of the Self-Care of coronary heart disease inventory (SC-CHDI). Res Nurs Health 40: 15-22, 2017.

15. Huang L, Zheng Y, Yuan X, Ma Y, Xie G, Wang W, Chen H and Shen L: Decreased frequencies and impaired functions of the $\mathrm{CD} 31^{+}$subpopulation in $\mathrm{T}_{\mathrm{reg}}$ cells associated with decreased FoxP3 expression and enhanced Treg cell defects in patients with coronary heart disease. Clin Exp Immunol 187: 441-454, 2017.

16. Tang SS, Xu S, Cheng J, Cai MY, Chen L, Liang LL, Yang XL, Chen C, Liu XG and Xiong XD: Two tagSNPs rs352493 and rs3760908 within SIRT6 gene are associated with the severity of coronary artery disease in a chinese han population. Dis Markers 2016: 1628041, 2016.

17. McLaren JE, Michael DR, Ashlin TG and Ramji DP: Cytokines, macrophage lipid metabolism and foam cells: Implications for cardiovascular disease therapy. Prog Lipid Res 50: 331-347, 2011.

18. Wu G, Li GB and Dai B: Association of KIF6 variant with lipid level and angiographic coronary artery disease events risk in the Han Chinese population. Molecules 17: 11269-11280, 2012.

19. Ntekim OE, Allard JS, Ngwa JS, Johnson AA, Castor C, Iyalomhe O, Fungwe TV, Ntekim CC and Obisesan T: Additive effects of capsaicin oleoresin, irbesartan and amlodipine besylate on the blood pressure of spontaneously hypertensive rats. J Med Plants Res 10: 468-478, 2016.

20. Strupp M: The results support the use of atorvastatin in elderly patients with recent stroke or TIA. Neurology 73: 817, 2009.

21. Xin H, Chen ZY, Lv XB, Liu S, Lian ZX and Cai SL: Serum secretory phospholipase A2-IIa (sPLA2-IIA) levels in patients surviving acute myocardial infarction. Eur Rev Med Pharmacol Sci 17: 999-1004, 2013.

22. Burstein B and Nattel S: Atrial fibrosis: Mechanisms and clinical relevance in atrial fibrillation. J Am Coll Cardiol 51: 802-809, 2008.

23. Rivard L and Khairy P: Mechanisms, clinical significance, and prevention of cognitive impairment in patients with atrial fibrillation. Can J Cardiol 33: 1556-1564, 2017.

24. Ikonomidis I and Michalakeas CA: Phospholipases in cardiovascular disease. In: Advances in Biochemistry in Health and Disease: Phospholipases in Health and Disease. Tappia PS and Dhalla NS (eds). Vol 10. Springer, New York, pp73-83, 2014.

25. Divchev D, Grothusen C, Luchtefeld M, Thoenes M, Onono F, Koch R, Drexler H and Schieffer B: Impact of a combined treatment of angiotensin II type 1 receptor blockade and 3-hydroxy-3-methyl-glutaryl-CoA-reductase inhibition on secretory phospholipase A2-type IIA and low density lipoprotein oxidation in patients with coronary artery disease. Eur Heart J 29: 1956-1965, 2008 .

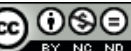

This work is licensed under a Creative Commons Attribution-NonCommercial-NoDerivatives 4.0 International (CC BY-NC-ND 4.0) License. 